\title{
Rectangular waveguides with two conventional and two superconducting walls
}

\author{
RAJ YALAMANCHILI†, ZHENG AN QIU† and \\ YEN-CHU WANG†
}

\begin{abstract}
The propagation properties of $\mathrm{TE}^{\mathrm{pm}}$ modes and their dispersion relations in rectangular waveguides with two conventional and two superconducting walls, derived by using the Meissner boundary conditions on the superconducting walls, are presented. In addition to recovering some previously known results, some novel results have been obtained: the cut-off wavelength of the dominant TE ${ }^{10}$ mode is greater than that of the conventional $\mathrm{TE}_{10}$ mode, and the tangential electric field and normal magnetic field for the dominant mode $\mathrm{TE}^{10}$ exist on the superconducting surfaces. Expressions for electromagnetic components, surface currents, attenuation coefficient, maximum transmitted power, dispersion and wave impedance are also presented.
\end{abstract}

\section{Introduction}

Over several years there has been considerable progress in the development of superconducting devices in the microwave and millimetre wave bands, for example, low- and high-temperature superconducting microwave filters, resonators, antennas, phase shifters, etc. Many superconducting electronic devices have been sought at liquid nitrogen temperatures (Nisenoff 1988, Van Duzer and Tuner 1981). The discovery of high- $T_{c}$ superconductors (HTS) has fundamentally changed the prospects of superconductive electronics. The low surface resistance of superconducting materials makes possible microwave devices and circuits with very high $Q$, low insertion loss and dispersion. Superconducting waveguides have been studied as low $T_{c}$ superconductors $(<18 \mathrm{~K}$ ) (Alaux and Wybouw 1976, Rohner 1978, Zepp et al. ' 1977, Fabre et al. 1981). Experimental and theoretical results have shown that HTS waveguides and transmission lines exhibit significantly better performance than that of their identical metallic counterparts (Wang et al. 1994, Yalamanchili et al. 1992).

The propagation properties of transverse electric (TE) modes in high- $T_{c}$ $\left(T_{c}>30 \mathrm{~K}\right)$ superconductor rectangular waveguides have recently been studied using HTS electromagnetic theory and Meissner boundary conditions which are consistent with the two-fluid model (Wang et al. 1994). The new boundary conditions give rise to different field solutions, hence new waveguide characteristics. This paper describes the propagation properties of TE modes in rectangular waveguides with two conventional and two superconducting walls (WGCSW) based on the theory established by Wang et al. (1994). This kind of waveguide has different characteristics from either HTS waveguides or conventional waveguides. The attenuation and dispersion are smaller than that of conventional waveguides. The dominant mode in the WGCSW is the TE ${ }^{10}$ mode, which is the same as conventional waveguides. An interesting property is that the cut-off wavelength of the dominant mode is greater

Received 18 June 1994; accepted 27 October 1994.

† Department of Electrical Engineering, Howard University, Washington, DC 20059 , U.S.A. 
than that of conventional waveguides. Therefore, it is easier to excite the dominant mode in the WGCSW. Since the WGCSW has two conventional broad walls and two superconducting walls (two simply-connected superconductors), it is easier to fabricate than a four-wall superconducting waveguide.

In section 2, the most important dispersion relation of WGCSW is derived along the $x$-direction from the wave equation and the Meissner boundary conditions on two walls $(x=0, x=a)$. Expressions for electromagnetic field components and surface currents, including the surface superconducting current $J_{m}$ and the surface normal current $J_{s n}$, based on two-fluid theory are given. Various parameters of the WGCSW are analysed and compared with conventional waveguides.

\section{Theory}

\subsection{Wave equation and numerical solutions of cut-off wavelength}

This section analyses a WGCSW with two narrow superconducting walls ( $x=0$ and $x=a)$ and two broad perfect or good conductor walls $(y=0$ and $y=b)$, as shown in Fig. 1. A rigorous formulation based on Maxwell's equations and Meissner boundary conditions is used to obtain the new electromagnetic field properties in the WGCSW.

The governing wave equation (Helmholtz equation) and the Meissner boundary conditions for the longitudinal magnetic field $H_{2}$ of the TE mode in an air-filled WGCSW are:

$$
\begin{gathered}
\nabla_{\mathrm{T}}^{2} H_{z}+k_{\mathrm{c}}^{2} H_{\mathrm{z}}=0 \\
\frac{\partial H_{z}}{\partial n}-\frac{1}{\lambda_{\mathrm{L}}} H_{z}=0 \quad \text { at } x=0, x=a \\
\frac{\partial H_{z}}{\partial n}=0 \quad \text { at } y=0, y=b
\end{gathered}
$$

where the operator $\nabla_{T}^{2}$ is the Laplacian operator in the transverse plane (i.e. $x y$-plane). Propagation in the $z$-direction is assumed. The parameters $a$ and $b$ are the

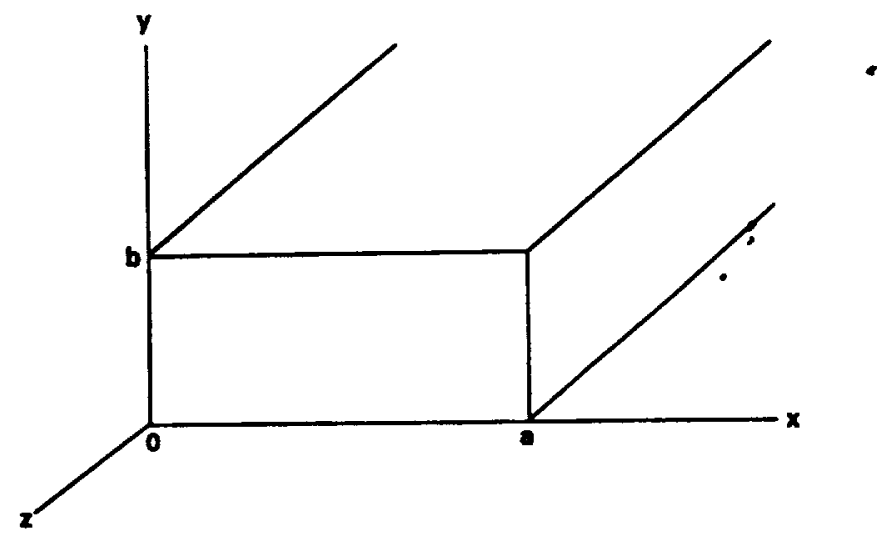

Figure 1. WGCSW with two narrow superconducting walls $(x=0, x=a)$ and two broad perfect or good conductor walls $(y=0, y=b)$. 
width and height of the WGCSW, respectively; $k_{\mathrm{c}}$ is the cut-off wavenumber; and $\lambda_{\mathrm{L}}$ is the London penetration depth, the value of which for HTS varies from $10^{-8} \mathrm{~m}$ to $10^{-7} \mathrm{~m}$ (Burns 1992). Equation ( $2 a$ ) is the Meissner boundary condition; $(2 b)$ is the boundary condition for the conventional wall. The important Meissner effect is that the fields do not vanish abruptly from the surface of a bulk superconductor, rather, they decay exponentially into the bulk. The penetration depth $\lambda_{\mathrm{L}}$ is the characteristic decay length of the magnetic field into a superconductor. It is obvious that when $\lambda_{L} \rightarrow \infty$, the second term of the Meissner boundary condition approaches zero, $(2 a)$ reduces to the boundary condition $\left({ }^{o} \mathrm{H}_{z} /{ }^{\circ} n\right)=0$ for the conventional waveguide. Therefore, the first term of $(2 a)$ is related to the properties of the WGCSW. Thus, the Meissner boundary conditions are relevant to the two-fluid superconducting model.

It is assumed that the general solution of the governing equation is

$$
H_{z}=\left(C_{1} \sin k_{x} x+C_{2} \sin k_{x} x\right) \cos k_{y} y
$$

where $C_{1}$ and $C_{2}$ are unknown coefficients; both cannot be equal to zero at the same time; $k_{x}$ and $k_{y}$ are the uknown wavenumbers along the $x$ and $y$ axes, respectively.

Substituting (3) into (2a) and (2b) obtains two sets of equations for $k_{x}$ and $k_{y}$ :

$$
\begin{gathered}
\left(k_{x} \sin k_{x} a-\frac{\cos k_{x} a}{\lambda_{\mathrm{L}}}\right) C_{1}-\left(k_{x} \cos k_{x} a+\frac{\sin k_{x} a}{\lambda_{\mathrm{L}}}\right) C_{2}=0 \\
\frac{1}{\lambda_{\mathrm{L}}} C_{1}-k_{x} C_{2}=0
\end{gathered}
$$

and

$$
k_{y}=\frac{m \pi}{b}, \quad m=0,1,2,3, \ldots
$$

For non-trivial solution conditions, the determinant of the coefficient matrix of $(4 a)$ and $(4 b)$ has to be equal to zero:

$$
\left|\begin{array}{ll}
\frac{1}{\lambda_{2}} & -k_{x} \\
k_{\mathrm{x}} \sin k_{x} a-\frac{\cos k_{x} a}{\lambda_{L}} & -k_{x} \cos k_{x} a-\frac{\sin k_{x} a}{\lambda_{L}}
\end{array}\right|=0 .
$$

Therefore, the one important dispersion relation in the transcendental equation is obtained from the above determinant as

$$
\tan k_{x} a=\frac{2 \lambda_{1} k_{x}}{\left(k_{x} \lambda_{L}\right)^{2}-1}
$$

The non-integer roots of this equation describe the dispersion relations along the $x$-axis as a function of London penetration depth and the dimensions of the WGCSW. A graphical method is used for the solution, as shown in Fig. 2, from which the cut-off features are obtained. 


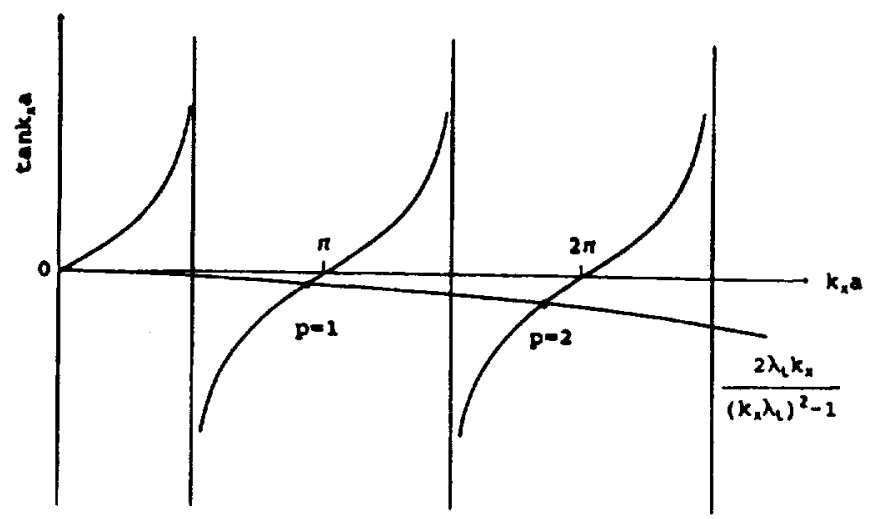

Figure 2. Graphical method of solving (7) to obtain the cut-off features $(p=1$ is the first root).

Substituting (4a) into (3),

$$
H_{z}=C_{1}\left(\cos k_{x} x+\frac{1}{k_{x} \lambda_{L}} \sin k_{x} x\right) \cos k_{y} y
$$

or

$$
H_{z}=C_{2}\left(k_{x} \lambda_{L} \cos k_{x} x+\sin k_{x} x\right) \cos k_{y} y
$$

It is obvious from (9) that $k_{x} \neq 0$, otherwise the solution becomes trivial. It is worth noting that owing to the non-integral nature, the mode indexes are determined by solving (7), which is derived from the Meissner boundary conditions on the two walls.

To avoid risk of confusion with the commonly used $\mathrm{TE}_{\mathrm{nm}}$, the superscripts $p m$ (the index $p$ is related to the superconducting walls) are used to designate the modes' in the WGCSW. In this case, the dominant mode is designated $\operatorname{TE}^{10}(p=1$ does not mean integer index along the $x$-axis, instead it is the first root of (7)). The above formula, however, does cover conventional waveguides in which $H_{z} \propto \cos k_{x} x \cos k_{y} y$ when $\lambda_{L} \rightarrow \infty$, as is evident from (8). There are no TE ${ }^{0 m}$ modes in the WGCSW, in which property it is quite different from conventional waveguides.

Substituting (3) into (1),

$$
k_{\mathrm{c}}^{2}=k_{x}^{2}+k_{y}^{2}
$$

Therefore, the cut-off wavelength $\lambda_{c}$ is obtained as

$$
i_{\mathrm{c}}=\frac{2 \pi}{k_{\mathrm{c}}}
$$

The numerical results of cut-off wavelengths for some $T^{p m}$ modes are listed in the Table. These results demonstrate that the cut-off wavelength of the dominant mode in the WGCSW $\left(\lambda_{c}^{10} \approx 4.06 \mathrm{~mm}\right.$ for $\left.a=2 \mathrm{~mm}\right)$ is greater than that of $T_{10}$ in a conventional waveguide with the same dimensions by a factor of 1.013, and the bandwidth is greater than an octave. 


\subsection{Field components and surface current distributions}

Field component expressions for the TE mode can be determined from the axial magnetic field $H_{3}$ by means of the relations between the transverse and axial components as follows.

From (9), let $B_{0}=k_{x} \lambda_{L}$ and $H_{0}=C_{2}$ for simplicity. Then the axial magnetic field component $H_{2}$ becomes

$$
H_{z}=H_{0}\left(B_{0} \cos k_{x} x+\sin k_{x} x\right) \cos k_{y} y
$$

where $H_{0}$ is an unknown coefficient depending on excitations, $B_{0}$ is a proportionality parameter which varies for different modes and penetration depths. The wave factor in the form of $\exp (j \omega t-\gamma z)$ is assumed. The real part $\alpha$ of the propagation constant $\gamma$ is the attenuation constant, the imaginary part $\beta$ is the phase constant and $\omega$ is the angular frequency. Other field components can be expressed as

$$
\begin{aligned}
& H_{x}=\frac{\mathrm{j} \beta k_{x}}{k_{\mathrm{c}}^{2}} H_{0}\left(B_{0} \sin k_{x} x-\cos k_{x} x\right) \cos k_{y} y \\
& H_{y}=\frac{\mathrm{j} \beta k_{y}}{k_{\mathrm{c}}^{2}} H_{0}\left(B_{0} \cos k_{x} x+\sin k_{x} x\right) \sin k_{y} y \\
& E_{x}=\frac{\mathrm{j} \omega \mu_{0} k_{y}}{k_{\mathrm{c}}^{2}} H_{0}\left(B_{0} \cos k_{x} x+\sin k_{x} x\right) \sin k_{y} y \\
& E_{y}=\frac{\mathrm{j} \omega \mu_{0} k_{x}}{k_{\mathrm{c}}^{2}} H_{0}\left(B_{0} \sin k_{x} x-\cos k_{x} x\right) \cos k_{y} y
\end{aligned}
$$

where $\mu_{0}$ is the permeability of the material, assumed to be that of free space.

The wave impedance $Z_{b}$ of the WGCSW is defined as

$$
Z_{\mathrm{b}}=\frac{E_{x}}{H_{y}}=-\frac{E_{y}}{H_{x}}=\frac{\omega \mu_{0}}{\beta}
$$

\begin{tabular}{cccccc}
\hline & \multicolumn{5}{c}{ Cut-off wavelength (mm) } \\
\cline { 2 - 6 } Modes & $a=1$ & \multicolumn{1}{c}{$a=2$} & \multicolumn{1}{c}{$a=4$} & \multicolumn{1}{c}{$a=8$} \\
\hline $\mathrm{TE}^{10}$ & $2.05(2.0) \dagger$ & $4.06(4.0)$ & $8.07(8.0)$ & $.16 .09(16.0)$ \\
$\mathrm{TE}^{20}$ & $1.02(1.0)$ & $2.04(2.0)$ & $4.07(4.0)$ & $8.04(8.0)$ \\
$\mathrm{TE}^{11}$ & $0.90(0.89)$ & $1.79(1.78)$ & $3.58(3.58)$ & $7.16(7.15)$ \\
$\mathrm{TE}^{21}$ & $0.70(0.70)$ & $1.42(1.41)$ & $2.83(2.82)$ & $5.66(5.65)$ \\
$\mathrm{TE}^{30}$ & $0.67(0.48)$ & $1.34(0.97)$ & $2.67(1.94)$ & $5.34(3.88)$ \\
$\mathrm{TE}^{31}$ & $0.56(0.44)$ & $1.11(0.89)$ & $2.22(1.79)$ & $4.44(3.58)$ \\
\hline
\end{tabular}

+ Values in parentheses are the corresponding cut-off wavelengths for the first six TE $_{m-m}$ modes in conventional waveguides.

Cut-off wavelengths for some TE ${ }^{p m}$ modes $\left(\lambda_{L}=10^{-7} \mathrm{~m}, a=2 b\right.$; for $\lambda_{L}=10^{-8} \mathrm{~m}$ there is little difference in the values). 
The instantaneous field expressions for the dominant $T E^{10}$ mode in the WGCSW can be obtained as:

$$
\begin{aligned}
& H_{z}=H_{0}\left(B_{0} \cos k_{x} x+\sin k_{x} x\right) \cos (\omega t-\beta z) \\
& H_{x}=\frac{\beta k_{x}}{k_{c}^{2}} H_{0}\left(B_{0} \sin k_{x} x-\cos k_{x} x\right) \sin (\omega t-\beta z) \\
& E_{y}=\frac{\omega \mu_{0} k_{x}}{k_{c}^{2}} H_{0}\left(-B_{0} \sin k_{x} x+\cos k_{x} x\right) \sin (\omega t-\beta z)
\end{aligned}
$$

where, $\beta=2 \pi / \lambda_{2}$ is the phase constant of the TE ${ }^{10}$ mode, and $\lambda_{2}$ is the waveguide wavelength of the TE ${ }^{10}$ mode. There are three field components in the WGCSW, as in conventional waveguides. Note that the superscript ' 10 ' for the dominant mode is omitted from here on unless otherwise stated.

From the above discussion, ( $15 c$ ) demonstrates clearly that, due to $B_{0}$ being very small, the space distribution of the electric field $E$, of the $\mathrm{TE}^{10}$ mode is proportional, significantly, to $\cos k_{x} x$ along the $x$-direction. This means that tangential electric fields exist on the superconducting surfaces $(x=0$ and $x=a)$ of the WGCSW. This novel property is completely different from that of a perfectly conducting conventional waveguide since, for $H_{3} \neq 0$ and for a finite surface impedance at microwave and millimetre wavelengths, the tangential components of $E$ on the superconducting walls are finite. The space distribution along the $y$-direction is the same as for conventional waveguides. For details of the field distributions see Fig. 3.

Equation ( $15 b)$ demonstrates that the space distribution of the magnetic field $H_{x}$ is largely proportional to $\cos k_{x} x$, which means that normal magnetic fields exist on the surfaces $(x=0$ and $x=a)$ (see Fig. 3). The above results demonstrate that the magnetic field lines are not continuous in the WGCSW (Orlando and Delin 1991), which is in agreement with the Meissner effect, but the magnetic flux density $B$ is continuous in and out of the WGCSW walls because $B=\mu_{0}(H+M)(M$ is the magnetization density in the walls and is not equal to zero). This also is a new property in the WGCSW, which does not exist in conventional-waveguides.

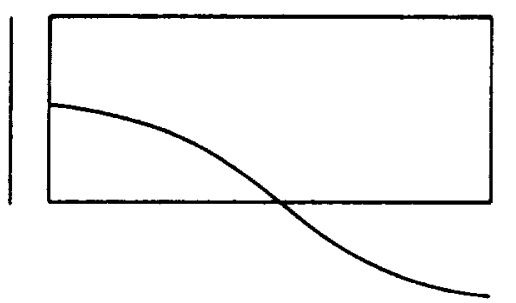

(a)

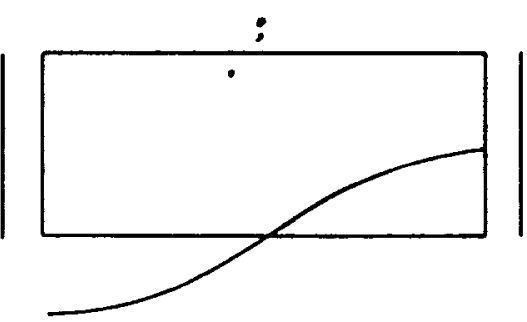

(b)

Figure 3. (a) Distribution of the electric field $E_{\text {; }}$ (b) distribution of the magnetic field $H_{x}$, for the TE ${ }^{10}$ mode. 
London's equations and the two-fluid model are used to investigate the current distribution on the surfaces $(x=0$ and $x=a)$. The total conduction current density consists of the superconducting current density $J_{2}$ and the normal electron density $J_{n}$ based on the two-fluid model. This model assumes that the conducting electrons in the WGCSW walls are divided into two categories. We use a different method to describe their distribution. The surface superconducting current $J_{w}$ due to superelectrons is calculated based on the London relation and appropriate boundary condition first. Then the normal surface current $J_{3 n}$ due to normal electrons and the losses is calculated using the approximate resistive boundary condition and the perturbation approach.

The superconducting current $J_{1}$ in two isolated walls in which each wall is considered as a simply-connected superconductor using a thin-film substrate is given as

$$
J_{2}=-\frac{1}{A} A
$$

This is London's relation, where $A=\mu_{0} \lambda_{\mathrm{L}}^{2}$, and $A$ (in $\mathrm{Tm}$ ) is the vector potential in the WGCSW. Let the vector potential inside the air-filled hollow region in the waveguide be $A_{\mathrm{a}}$ (subscript a indicates the air-filled WGCSW). This satisfies the following equations:

$$
\begin{gathered}
\nabla \chi_{1} A_{2}=B \\
E=\frac{k^{2} A_{2}+\nabla \nabla \cdot A_{2}}{j \omega \mu_{0} \varepsilon_{0}}
\end{gathered}
$$

where $\varepsilon_{0}$ is the permittivity of the material.

Thus, the London relation guarantees that the vector potential is a 'real field' that is completely specified. Equation (18) is called Lorentz's gauge, which gives rise to the relation between the electric field $E$ and the vector fotential $A_{\mathbf{a}}$ in the air region.

Before solving the current distribution problem, it is important to determine the boundary condition at a boundary between the two media (here, the superconducting medium and air for walls $x=0$ and $x=a$; the perfect conductor and air for walls $y=0$ and $y=b)$. The boundary condition on the tangential components of fields $(J$, and $A_{2}$ ) for the simply-connected superconductor is

$$
J_{\mathrm{s}}=-\frac{1}{\Lambda} A_{\mathrm{at}}
$$

where the subscript 'at' denotes the component tangential to the boundary in the air or the applied field region; 'st' denotes the component of supercurrent density tangential to the boundary in the HTS. Note that $J_{u}$ is the bulk current. Once $A_{a}$ is known, the current density $J_{31}$ and the surface supercurrent density $J_{\text {sat }}$ are readily calculated. The solution satisfying (17) and (18) is obtained from $B$ as follows: 


$$
\begin{aligned}
A_{\mathrm{az}}= & A_{0}\left(B_{0} \sin k_{x} x+\cos k_{x} x\right) \sin k_{y} y \\
A_{\mathrm{ax}}= & \frac{\mathrm{j} A_{0} k_{x}}{\beta} H_{0}\left(B_{0} \cos k_{x} x-\sin k_{x} x\right) \sin k_{y} y \\
& -\frac{\mu_{0} k_{y}}{k_{\mathrm{c}}^{2}} H_{0}\left(B_{0} \cos k_{x} x+\sin k_{x} x\right) \sin k_{x} y \\
A_{\mathrm{a} y}= & \frac{\mathrm{j} A_{0} k_{y}}{\beta}\left(B_{0} \sin k_{x} x+\cos k_{x} x\right) \cos k_{y} y \\
& +\frac{\mu_{0} k_{x}}{k_{\mathrm{c}}^{2}} H_{0}\left(B_{0} \sin k_{x} x-\cos k_{x} x\right) \cos k_{y} y
\end{aligned}
$$

The surface supercurrent distributions on the isolated walls $(x=0$ and $x=a)$ are obtained from (19) as follows:

$(x=0$ plane $)$

$$
\begin{aligned}
J_{\mathrm{set}} & =J_{\mathrm{sy}} a_{y}+J_{\mathrm{szz}} a_{z} \\
& \approx \frac{\lambda_{\mathrm{L}}}{\Lambda}\left[\frac{\mu_{0} k_{x}}{k_{\mathrm{c}}^{2}} H_{0} \cos k_{y} y-\frac{\mathrm{j} A_{0} k_{y}}{\beta} \cos k_{y} y\right] a_{y}-\frac{A_{0} \dot{\lambda}}{\Lambda} \sin k_{y} y a_{z}
\end{aligned}
$$

( $x=a$ plane $)$

$$
\begin{aligned}
J_{y+1} & =J_{s y} a_{y}+J_{s z z} a_{z} \\
& \approx \frac{\lambda_{\mathrm{L}}}{A}\left[\frac{\mu_{0} k_{x}}{k_{\mathrm{e}}^{2}} H_{0} \cos k_{y} y-\frac{\mathrm{j} A_{0} k_{y}}{\beta} \cos k_{y} y\right] a_{y}+\frac{A_{0} \dot{\lambda}}{A} \sin k_{y} y a_{z}
\end{aligned}
$$

where $J_{2 a y}$ and $J_{3 a z}$ are the surface supercurrent magnitudes on the walls along the $y$ and $z$-directions, respectively. Consider, here, that the superconducting current decays exponentially in the walls, so the current is mainly carried by the superconductor surface adjacent to the air in the WGCSW. It is easy to see that the surface supercurrent and bulk current are very large because the ámplitude of $J_{w a}$ is proportional to $1 / \lambda_{L}\left(\Lambda=\mu_{0} \lambda_{L}^{2}\right)$, which is of the order of $10^{7}$.

All high- $T_{\mathrm{c}}$ materials are type II superconductors. In the Meissner phase, it is assumed that the critical field $H_{\mathrm{cl}} \approx 7.7$ to $385 \mathrm{~A} \mathrm{~m}^{-1}$ (depending on temperature) for a HTS sample of $\mathrm{YBa}_{2} \mathrm{Cu}_{3} \mathrm{O}_{y}\left(88.2 \mathrm{~K}, \boldsymbol{H}_{\mathrm{c}}^{\perp}\right.$ axis) (Wu and Sridhar 1990), so if $H_{0} \approx 13$ to $660 \mathrm{~A} \mathrm{~m}^{-1}$, then the theoretical values of supercurrent density are estimated to be about $10^{6-8} \mathrm{~A} \mathrm{~cm}^{-2}$ which is in excellent agreement with other data (Burns 1992, Heinen et al. 1991, Miranda et al. 1991, Levenson et al. 1991). The distributions of surface supercurrent and normal current on the $x=0$ and $x=a$ 
planes of the WGCSW are shown in Fig. 4. (Note that the distributions of the real and imaginary parts of the surface supercurrent of the WGCSW are the same.)

The surface normal current distribution at its boundaries can be obtained using the approximate resistive boundary condition $\boldsymbol{n} \times \boldsymbol{H}=\boldsymbol{J}_{\mathrm{sn}}$ (Senior 1975) as given by (on the $y=0$ plane)

$$
\begin{aligned}
J_{\mathrm{sn}} & =J_{\mathrm{sn} x} a_{x}+J_{\mathrm{snz}} a_{z} \\
& =H_{0}\left(B_{0} \cos k_{x} x+\sin k_{x} x\right) a_{x}-\frac{\mathrm{j} \beta k_{x}}{k_{c}^{2}} H_{0}\left(B_{0} \sin k_{x} x-\cos k_{x} x\right) a_{z}
\end{aligned}
$$

(on the $y=b$ plane)

$$
\begin{aligned}
J_{\mathrm{sn}}= & J_{\mathrm{sn} x} a_{x}+J_{\mathrm{snz}} a_{z} \\
= & -H_{0} \cos k_{y} b\left(B_{0} \cos k_{x} x+\sin k_{x} x\right) a_{x} \\
& +\frac{\mathrm{j} \beta k_{x}}{k_{\mathrm{c}}^{2}} H_{0} \cos k_{y} b\left(B_{0} \sin k_{x} x-\cos k_{x} x\right) a_{z}
\end{aligned}
$$

(on the $x=0$ plane)

$$
J_{\mathrm{zan}}=J_{\mathrm{sa}, a_{y}}+J_{\mathrm{saz}} a_{z}=H_{0} B_{0} \cos k_{y} y a_{y}-\frac{\mathrm{j} \beta k_{y}}{k_{\mathrm{c}}^{2}} H_{0} B_{0} \sin k_{\mathrm{y}} y a_{z}
$$

(on the $x=a$ plane)

$$
\begin{aligned}
J_{s a}= & J_{\text {syy }} a_{y}+J_{\text {snz }} a_{z} \\
= & -H_{0}\left(B_{0} \cos k_{x} a+\sin k_{x} a\right) \cos k_{y} y a_{y} \\
& +\frac{\mathrm{j} \beta k_{y}}{k_{\mathrm{c}}^{2}} H_{0}\left(B_{0} \cos k_{x} a-\sin k_{x} a\right) \sin k_{y} y a_{z}
\end{aligned}
$$

where $J_{z n y}$ and $J_{s n z}$ are the surface normal current magnitudes on the walls along the $y$-and $z$-directions, respectively. It is easy to see that the distributions of the surface normal currents on the walls $x=0$ and $x=a$ are the same as those of the surface supercurrents on the walls, which is the result of the two-fluid model. But the distributions of current on the $y=0$ and $y=a$ surfaces differ from conventional waveguides due to different electromagnetic field distributions. The losses of the WGCSW come from the surface normal current flowing through the small surface

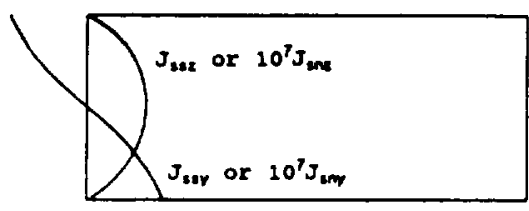

(a)

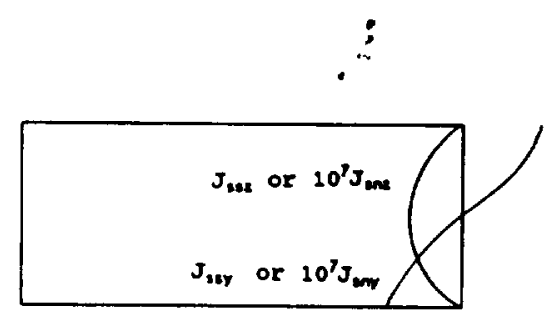

(b)

Figure 4. Distribution of surface supercurrent and surface normal current for the $\mathrm{TE}^{10}$ mode on the walls $(a) x=0,(b) x=a$. 
impedance. It is predictable that the losses are small because $B_{0}$ is very small. The distributions of surface current on the WGCSW planes $y=0$ and $y=b$ are shown in Fig. 5.

\subsection{Attenuation coefficient}

The attenuation caused by two superconducting walls and two conventional walls can be calculated by the perturbation technique as given approximately by

$$
\alpha^{10} \approx 3.07 \times 10^{-7} \frac{f_{\mathrm{c}}\left[R_{\mathrm{s}}\left(\frac{f_{\mathrm{c}}}{f}\right)^{2}+R_{\mathrm{n}}\right]}{\left[1-\left(\frac{f_{\mathrm{c}}}{f}\right)^{2}\right]^{1 / 2}} \mathrm{~dB} \mathrm{km^{-1 }}
$$

where $f_{\mathrm{c}}$ is the cut-off frequency of the TE ${ }^{10}$ mode, $R_{\mathrm{g}}$ and $R_{\mathrm{n}}$ are the surface resistances of HTS and metal walls, respectively, and $\lambda_{L}$ is in millimetres. Note that the attenuation is not only proportional to $f_{c}^{3}$ because $R_{\mathrm{a}} \propto f^{2}$ (Romanofsky and Bhasin 1992), but also proportional to $\lambda_{\mathrm{L}}^{2}\left(R_{\mathrm{n}} \propto f^{1 / 2}\right)$. The curve of attenuation coefficient $\alpha$ versus frequency $f$ is shown in Fig. 6 in which $R_{s} \approx 5.0 \times 10^{-28} f^{2} \Omega$, $R_{\mathrm{n}} \approx 2.6 \times 10^{-7} f^{1 / 2} \Omega(\mathrm{Cu})$, and $a=2 b=2 \mathrm{~mm}$. As far as the attenuation is concerned, it is greater than that of high- $\mathrm{T}_{\mathrm{c}}$ superconducting waveguides, but smaller than that of conventional waveguides.

Losses in the WGCSW are generated from three sources: superconductor loss, metal loss and dielectric loss. A typical superconducting waveguide used in microwave and millimetre-wave circuits uses low-loss dielectrics or free space, so the dominant loss mechanisms are the superconductor loss and metal loss.

The superconductor loss is caused by the current of normal electrons in the superconducting walls based on the two-fluid model theory. The paired superconducting electrons are dissipationless because they cannot be scattered without breaking pairs. The microwave surface resistance of superconducting films has been found to be as low as a few microohms. These surface resistance values are lower than those for copper at the same temperature by a factor of several tens. Such $a$ surface resistance gives a small power loss. If the penetration depth $\lambda_{L}=3.6 \times 10^{-7} \mathrm{~m}$ for the case of conduction along the c-axis and the surface resistance is equal to $10.3 \mathrm{~m} \Omega(60 \mathrm{~K})$ at $14.567 \mathrm{GHz}$ (How et al. 1992), the attenuation coefficient is found to be about $0.9 \mathrm{~dB} \mathrm{~m}^{-1}$ for $R_{\mathrm{n}} \approx 30 \mathrm{~m} \Omega$. The metal loss is the same as for conventional waveguides.

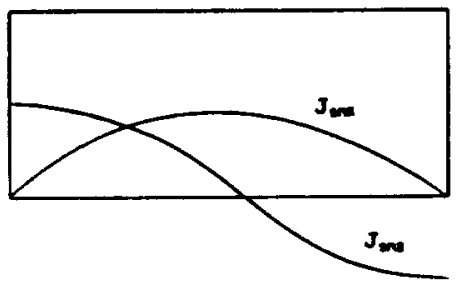

(a)

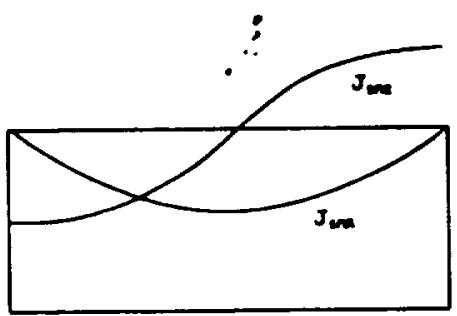

(b)

Figure 5. Distribution of surface current for the $\operatorname{TE}^{10}$ mode on the walls $(a) y=0,(b) y=b$. 


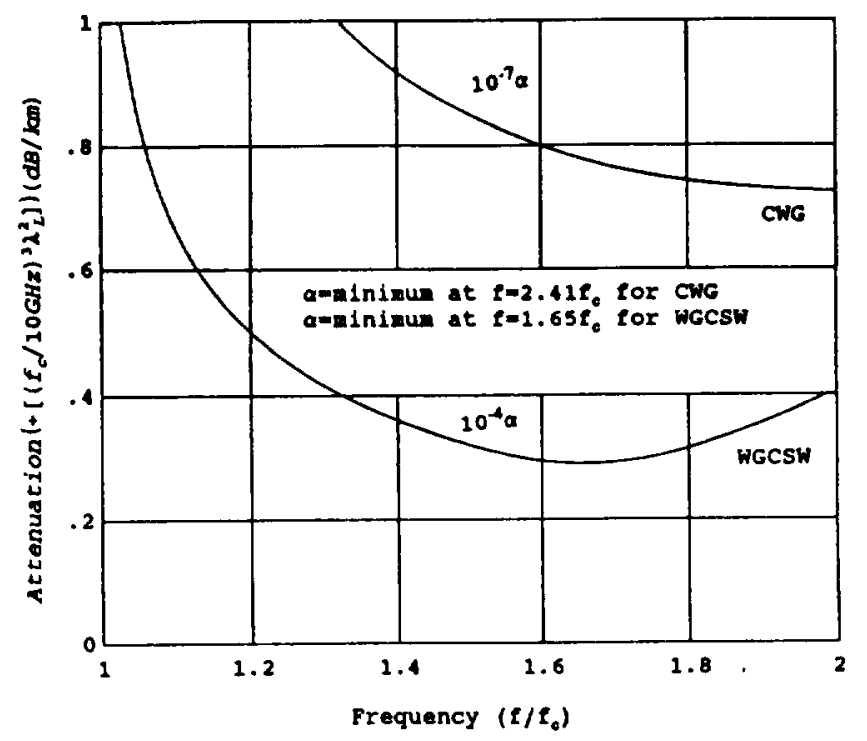

Figure 6. Attenuation due to wall losses versus frequency $\left(\lambda_{L}=10^{-7} \mathrm{~m}\right)$. CWG, conventional waveguide; WGCSW, waveguide with two conventional and two superconducting walls.

\subsection{Phase velocity, group velocity and phase dispersion}

Superconductors have a frequency-independent penetration depth in the terahertz range, which determines field penetration into the material, rather than a frequency-dependent skin depth as for normal conductors. This means that superconductors introduce practically no dispersion into a microwave circuit. Dispersion in the WGCSW, if any, is due to the frequency dependence of the attenuation caused by the two broad walls. Dispersion caused by the frequency dependence of the phase velocity is almost the same as for conventional waveguides. The phase velocity $v_{f}$ and the group velocity $v_{a}$ of the $\mathrm{TE}^{10}$ mode can be shown to be given by

$$
\begin{aligned}
& v_{\mathrm{p}}=\frac{\omega}{\beta}=\frac{c}{\left[1-\left(\frac{f_{\mathrm{c}}}{f}\right)^{2}\right]^{1 / 2}} \\
& v_{\mathrm{g}}=\frac{\mathrm{d} \omega}{\mathrm{d} \beta}=c\left(1-\left(\frac{f_{\mathrm{c}}}{f}\right)^{2}\right)^{1 / 2} \quad \therefore
\end{aligned}
$$

where $c$ is the speed of light in free space.

Our work shows that the phase velocity (group velocity) of the WGCSW for the dominant TE ${ }^{10}$ mode is smaller (greater) (by about $1.4 \%$ ) than that of conventional waveguides with the same dimensions and frequency because $f_{c}$ is smaller than that for conventional waveguides. Therefore, the dispersion produced for the same geometry is small. In summary, the signal distortion due to dispersion in the WGCSW is reduced dramatically by two superconducting walls. 


\subsection{Maximum transmilted power (dominant mode)}

The maximum transmitted power in the WGCSW is given by

$$
P_{\max }=\frac{a b}{4 \eta_{0}}\left|E_{\max }\right|\left[1-\left(\frac{f_{\mathrm{c}}}{t}\right)^{2}\right]^{1 / 2}
$$

where $\eta_{0}$ is the intrinsic impedance of the medium inside the waveguide, and the mangitude of the electric field intensity $\left|E_{\max }\right|$ is given from $(15 c)$ as

$$
\left|E_{\max }\right|=\left|H_{0}\right| \frac{\omega \mu_{0}}{k_{\mathrm{c}}}
$$

In the Meissner phase, the maximum electric field intensity and power in the WGCSW are smaller than that of conventional waveguides $\left(29 \mathrm{kV} \mathrm{cm}^{-1}\right.$ and $\left.11 \mathrm{~kW}\right)$ because $H_{0}$ is very small. For example, they are about $60 \mathrm{~V} \mathrm{~cm}^{-1}$ and $26 \mathrm{~mW}$ for $H_{\mathrm{c} 1} \approx 77 \mathrm{~mA} \mathrm{~cm}^{-1}$, and $3 \mathrm{kV} \mathrm{cm}^{-1}, 64 \mathrm{~W}$ for $H_{\mathrm{c} 1} \approx 3.85 \mathrm{~A} \mathrm{~cm}^{-1}$, respectively. It is clear from (26) and (27) that the transmitted power and maximum electric field are dependent on the London penetration depth $\left(k_{\mathrm{c}} \propto 1 / \lambda_{L}\right)$. The larger the London penetration depth, the larger is the transmitted power and maximum electric field. It should be noted that the penetration depth also increases with temperature, which means that more power is carried by the WGCSW.

\section{Conclusions}

A theoretical analysis for a waveguide with two conventional and two superconducting walls has been presented. It is based on high- $T_{c}$ superconducting electromagnetic theory and Meissner boundary conditions. The important implications of this paper are:

(a) the bandwidth of the WGCSW is greater than that of conventional metallic waveguides with the same dimensions;

(b) the tangential electric field and normal magnetic field of the TE ${ }^{10}$ mode are finite on the inner surfaces of the walls $(x=0$ and $x=a)$, which is in agreement with Wang et al. (1994);

(c) the magnetic field lines are not continuous in the WGCSW, which is the result of Meissner effect;

(d) the surface supercurrents are very large and the surface normal currents are very small, so the attenuation coefficient of the WGCSW is smaller than that of conventional waveguides by about $10^{2}$ to $10^{3}$ times for different surface resistance and penetration depth-the main loss mechanism is metal loss due to the two broad walls;

(e) in the Meissner phase, the maximum electric field intensity and transmitted power are smaller than those of conventional waveguides because the critical field $\left(H_{\mathrm{c} 1}\right)$ is very small;

$(f)$ dispersion is small compared with conventional waveguides;

(g) the wave impedance $Z_{b}$ of the WGCSW has the same expression as for conventional waveguides. 


\section{ACKNOWLEDGMENT}

This work was supported by NASA NAG5-1049.

\section{REFERENCES}

Alaux, A., and Wybouw, M., 1976, Propagation of even (EH) mode in superconducting elliptic waveguide. Canadian Journal of Physics, 54, 1488-1492.

BurNs, G., 1992, High-Temperature Superconductivity, An Introduction (New York: Academic Press).

FABRE, G., WiCK, A., and ZePP, G., 1981, Propagation of an electromagnetic wave on the surface of a conductor or superconductor with a circular cross-section. Canadian Journal of Physics, 59, 902-913.

Heinen, V. O., Bhasin, K. B., and Long, K. J., 1991, Emerging applications of hightemperature superconductors for space communications. Third Annual Digest, Solid State Technology Branch of NASA Lewis Research Center, pp. 101-109.

how, H., Seed, R. G., Vittoria, C., Chrisey, D. B., Horwitz, J. S., Carosella, C., and FOLEN, V., 1992, Microwave characteristics of high $T_{\mathrm{c}}$ superconducting coplanar waveguide resonator. IEEE Transactions on Microwave Theory and Technology, 40, 1668-1672.

Levenson, L. L., Stan, M., and Bhasin, K. B., 1991, Properties of large area $\mathrm{ErBa}_{2} \mathrm{Cu}_{3} \mathrm{O}_{7-x}$ thin films deposited by ionized cluster beams. Third Annual Digest, Solid State Technology Branch of NASA Lewis Research Center, pp. 127-130.

Miranda, F. A., Gordon, W. L., Bhasin, K. B., and Warner, J. D., 1991, Millimeter-wave surface resistance of laser-ablated $\mathrm{YBa}_{2} \mathrm{Cu}_{3} \mathrm{O}_{7-\phi}$ superconducting films. Third Annual Digest, Solid State Technology Branch of NASA Lewis Research Center, pp. 149-151.

NisenOFF, M., 1988, Superconducting electronics: current status and future prospects. Cryogenics, 28, 47-56.

Orlando, T. P., and Deuin, K. A., 1991, Foundations of Applied Superconductivity (Wokingham, U.K.: Addison-Wesley).

ROHNER, P. G., 1978, Possibility of low loss transmitting of RF-power with superconducting waveguide cables. Kerntechnik, 20, 489-493.

Romanofsky, R. R., and BhasiN, K. B., 1992, High temperature superconductor analog electronics for millimeter-wavelength communications. Fourth Annual Digest, Solid State Technology Branch of NASA Lewis Research Center, pp. 137-142.

SENIOR, T. B. A., 1975, Half plane edge diffraction. Radio Science, 10, 645-650.

VAN DUZER, T., and TURNER, C. W., 1981, Principles of Superconductive Devices and Circuits (New York: Elsevier).

Wang, Y.-C., QIU, Z. A., AND Yalamanchili, R., 1994, Meissner model of superconducting rectangular waveguides. International Journal of Electronics, 76, 1151-1171.

Wu, D. H., and SRidHrR, S., 1990, Pinning forces and lower critical fields in $\mathrm{YBa}_{2} \mathrm{Cu}_{3} \mathrm{O}_{4}$ crystals: temperature dependence and anisotropy. Physics Review Letters, 65, 2074-2077.

Yalamanchill, R., QIU, Z. A., and WANG, Y.-C., 1992, Review of microwave distributed superconducting vortex-flow transistor amplifier. International Journal of Electronics, 73, 585-604.

ZEPP, G., WICK, A, and FABRE, G., 1977, Influence of curvature in superconducting waveguides with circular cross-section. Canadian Journal of Physics, 55, 1551-1560. 



\section{ATTACHMENT B}


